\title{
Comparison of Initial Partitioning Methods for Multilevel Direct k-way Graph Partitioning with Fixed Vertices ${ }^{2}$
}

\author{
Maria Predari ${ }^{\mathrm{a}, *}$, Aurélien Esnard $^{\mathrm{a}}$, Jean Roman $^{\mathrm{b}}$ \\ ${ }^{a}$ Univ. of Bordeaux, Inria, CNRS (LaBRI UMR 5800) F33400 Talence, France \\ ${ }^{b}$ Inria, Bordeaux INP, CNRS (LaBRI UMR 5800), F33400 Talence, France
}

\begin{abstract}
In scientific computing, load balancing is a crucial step conditioning the performance of large-scale applications. In this case, an efficient decomposition of the workload to a number of processors is highly necessary. A common approach to solve this problem is to use graph representation and perform a graph partitioning in $k$-parts using the multilevel framework and the recursive bisection (RB) paradigm. However, in graph instances where fixed vertices are used to model additional constraints, RB often produces partitions of poor quality.

In this paper, we investigate the difficulties of $\mathrm{RB}$ to handle fixed vertices and we compare its results with two different alternatives. The first one, called KGGGP is a direct $k$-way greedy graph growing partitioning that properly handles fixed vertices while the second one, introduced in $\mathrm{KPATOH}$, uses RB and a post-processing technique to correct the obtained partition. Finally, experimental results on graphs that represent real-life numerical simulations show that both alternative methods provide improved partitions compared to RB. Keywords: high-performance computing ; graph partitioning ; fixed vertex ; multilevel framework; parallel simulations.
\end{abstract}

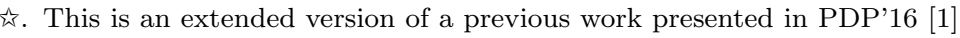

*. Corresponding author

Email addresses: maria.predari@inria.fr (Maria Predari), aurelien.esnard@labri.fr (Aurélien Esnard), jean.roman@inria.fr (Jean Roman)

Preprint submitted to Journal of ${ }^{A} T_{E} X$ Templates

3 février 2017 


\section{Introduction}

The increasing complexity of modern applications often dictates a decomposition of the computational load in order to ensure high performance. In other words, when parallel simulations are executed on large-scale systems, a distribu5 tion of their workload to a number of available processors is essential. Finding such a decomposition corresponds to the load balancing of a parallel application and results in a substantial minimization of the overall execution time.

In literature, many combinatorial problems such as the one described above can be solved in terms of graph modeling [2, 3, 4. More precisely a vertex of a graph represents a basic computation while an edge represents a dependency between two computations. Besides, each vertex has a weight proportional to the computation's cost, while each edge has a weight representing the communication costs between computations. Therefore, to balance the load of an application among $k$ processors, one may perform a graph partitioning in

${ }_{15} k$ parts of roughly equal size such that a minimal number of edges connects vertices in different parts. Nowadays, the most common approach to solve the graph partitioning problem is based on the multilevel approach to compress the problem and on the recursive-bisection heuristic to solve it on a smaller instance. Consequently, graph partitioning appears as a fundamental technique for 20 parallel computing.

However, in many applications the classic graph partitioning often fails to capture the true essence of the problem. In such settings, the model can be improved by including the "desire" of each vertex to be present in a certain part at the final solution. This idea has already been expressed in the form

25 of two problems. The first one is the skew graph partitioning that introduces a penalty function in the classic objective formula to represent the notion of 
desire [5]. During partitioning the desire function should be satisfied as an additional constraint to the classic ones.

In this paper, we focus on the second formulation which is a variant of the classic graph partitioning problem, the graph partitioning problem with initially fixed vertices. This modified instance of graph partitioning typically appears when the underlying application imposes strict constraints on the assignment of computations to specific processors. For instance, an application may have an a priori knowledge on data locality for certain computations. This information 35 can be modeled with the presence of fixed vertices in the initial graph, and may guide the partitioning procedure into reaching solutions of better quality. In particular, a fixed vertex is a vertex whose part assignment is pre-defined as part of the input description and shall not move throughout the partitioning. On the other hand, vertices that have no particular reason to be in a certain 40 part are referred to as free and may move to any part during partitioning.

In scientific computing, a well-known example where the fixed vertex paradigm occurs is the load balancing of adaptive scientific computations [5]. In such applications, the discretisation of the computational domain changes irregularly at run-time, leading to imbalanced load even for initially well-balanced simula45 tions. The above feature gives rise to the repartitioning problem that addresses the difficulties of maintaining a dynamically changing workload. The additional requirement of repartitioning is to minimize the migration volume of moving data among processors from a former partition to a new one. In this case, the graph is enriched with one fixed vertex per part, along with migration edges connecting each fixed vertex to all free vertices of its respective part. Thus, a good approach to solve the repartitioning problem is to use fixed vertices to model the additional constraint and perform a biased partitioning of the enriched graph, which minimizes migration costs, along with regular communication 
costs [6, 7].

Following, we consider the problem of load balancing for multi-physics, multiscales or multi-phase simulations that emerge in various areas such as computational physics, or hydrology and material science. Multi-physics simulations use coupled models in order to solve complex phenomena. Such an example is a hybrid particle/mesh transient dynamics simulation used to model car crashes and underwater explosions 8 . In such settings, acquiring a load decomposition that simultaneously balance both mesh and particle portions of the computation is challenging. Additionally, multi-phase simulations contain different computational phases separated with global synchronization points. In general, the amount of computations performed by each element of the mesh is different for different phases, leading to complex load balancing.

Note that the traditional graph partitioning model is not effective in such computations, and other strategies have been developed to address this problem. One approach is to distribute the overall computations of a multi-physics simulation by performing two different partitionings (a two-step partitioning) and then combine them together during a communication step [8, 9]. Obviously this approach has a major disadvantage, that is, data must be redistributed between the two partitions at every time-step of the simulation.

A solution to this problem is to introduce fixed vertices in order to use the results of the first phase/partitioning as an influence for the result of the second phase/partitioning. Therefore, fixed vertices may guide the partitioning to better solutions while the data re-distribution among different phases/partitionings may be avoided. Similar solutions have been proposed in [10, 11] in the context of multi-phase and coupled simulations respectively.

Note that another common approach for the load balancing of such simulations is based on the multi-constraint graph partitioning where multiple weights 
associated with the computational domain are simultaneously balanced [12, 13]. However we believe that this approach has an important limitation. More precisely, respecting multiple constraints is not straightforward especially when the number of parts increases and often results in highly imbalanced solutions [?]. Under this context, the graph partitioning with fixed vertices may be an important venue for the load balancing of large-scale multi-physics simulations.

Finally, graph partitioning with initial fixed vertices may be also used for non-numerical applications, such as the top-down placement technique [14] used in the integrated circuit design. In this case related studies [14, 15] suggest that the presence of fixed vertices represents more accurately additional positioning information, such as the locations of external pin connections, or the probable locations of certain cells in the final placement. As a result fixed vertices can direct the development of parts towards a better solution.

\subsection{Issues of Recursive Bisection in Partitioning with Fixed Vertices}

The motivation behind our study comes from the observation that RB based algorithms produce partitions of lower quality when the fixed vertex paradigm is involved, a remark initially shared in [16. Here, we illustrate a simple but compelling example that exhibits the partitioning issues of RB while in Section 3 we attempt to further explain this behavior.

For this example, we use a grid graph with an initial part numbering of fixed vertices such that vertices near the corners are assigned to four different parts following the scheme in Figure 1a. We consider the rest of the vertices as free (part -1) and we partition the graph in four parts. For the partitioning we use two different methods implemented within the same multilevel framework and tuned with the same parameters. To make the comparison of the partitioning phase easier, the refinement algorithms have been disabled for both methods. In Figure 1b we present the result obtained by the RB method (implemented 
by $\mathrm{ScOTCH}$ ), while in $1 \mathrm{c}$, we present the one obtained by KGGGP.

One may clearly see that under this fixed vertex scheme the partitioning quality of RB is considerably worse than that of KGGGP. We believe that the poor quality of RB is due to the way fixed vertices are pre-assigned to parts. More precisely, there is a conflict of the additional constraints imposed by fixed vertices and the inherent constraints of RB. Note that this example is a particular case of "bad" fixed vertex scheme but it clearly indicates the limitations of $\mathrm{RB}$. Indeed, $\mathrm{RB}$ is not robust when fixed vertices are included in the graph and may lead to highly degraded solutions. On the other hand, a method that is based on $k$-way direct graph growing techniques (for example the KGGGP method) could be more suitable for such problems.

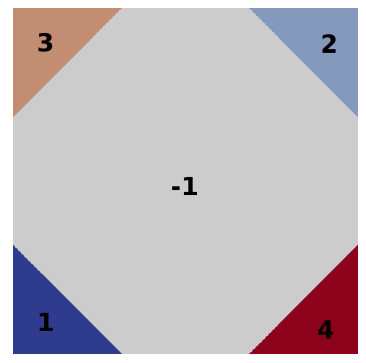

(a) Initial fixed vertices.

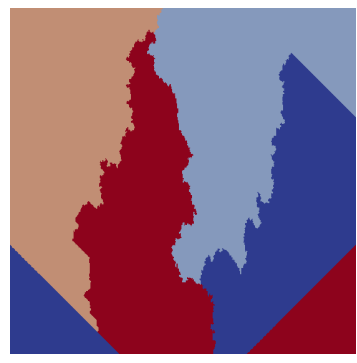

(b) RB.

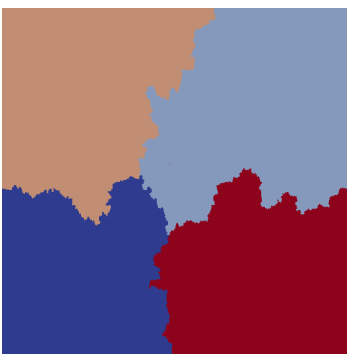

(c) KGGGP.

Figure 1: Given initial fixed vertices, comparison of two different partitioning methods (RB and KGGGP). Results of a 4-way partition of a $1000 \times 1000$ grid graph : the RB method fails to extend an initial partition while the KGGGP method succeeds.

The above example is just an illustration of the problematic behavior of RB methods with initial fixed vertices. Further experiments that confirm our observations follow in Section 6 .

\subsection{Contributions}

The main contribution of this paper is a extensive study of the graph partitioning problem with initially fixed vertices and a systematic comparison of different partitioning algorithms that find such solutions. This problem has not 
been thoroughly studied, even though it can be found in many scientific areas, as shown earlier in Section 1. Under this context, we compare the KGGGP method, presented by the authors in [1], with the state-of-the-art RB method and the RBBGM algorithm previously introduced in $\mathrm{KPATOH}$ [16. The comparison focuses on the edgecut quality and runtime efficiency when initially fixed vertices occur in the partitioning. Note that, as KPAToH is not publicly available, we provide a new implementation of the RBBGM method for graph structures. This allows a fair comparison of all methods since the overhead introduced by feeding edges to a hypergraph tool (KPATOH) is avoided. Additionally, using our own implementation for both approaches removes the dissimilarities that arise because of different coding practices. Finally, we enrich the experimental section of our preliminary work in 1] with a larger, highly diverse collection of graphs that show that both alternative methods provide improved partitions compared to RB.

The rest of the paper is organized as follows. In Section 2 we start by giving some background definitions and we present the state-of-the-art partitioning techniques as well as existing work for partitioning with fixed vertices. Then, in Section 3, we explain how RB works with fixed vertices and why it sometimes fails to properly solve such problems. In Section 4, we review in details the RBBGM method and in Section 5 we present the KGGGP algorithm. We confirm our observations in Section 6 presenting experiments performed on graphs that represent real numerical applications. Finally, we conclude our results in Section 7 .

\section{Background Definitions \& Related Work}

In this section, we first present useful definitions and formal statements concerning the partitioning problem in general and then we review existing 
studies about the graph partitioning problem with fixed vertices.

\subsection{Graph Definitions}

Consider a graph $G=(V, E)$ where $V$ is the set of vertices and $E$ is the set of edges. Each vertex $u \in V$ has a weight $w(u)$ representing its computational load, while each edge $e \in E$ has a weight $n(e)$ representing communication costs between different computations. A partition of a graph $G$ is an ensemble of $k$ vertex subsets, $P=\left(V_{1}, V_{2}, \ldots, V_{k}\right)$, where : i) each part $V_{i}, 1 \leq i \leq k$ is a non empty subset of $V$, ii) parts are pairwise disjoint $\left(V_{i} \cap V_{j}=\emptyset\right.$ for all $\left.1 \leq i, j \leq k\right)$ and iii) the union of all $k$ parts is equal to $V$. Given a vertex $v$ mapped to part $V_{i}$, we note $P[v]=i$ as its part number. The initial part numbering of free vertices is -1 while that of fixed vertices corresponds to their predefined part assignment.

Hence, the graph partitioning problem with edge separator can be defined as the optimization problem of dividing a graph into $k$ parts, $P=\left(V_{1}, V_{2}, \ldots, V_{k}\right)$, such that $P$ minimizes the total edgecut $: \min \sum_{e \in \mathcal{F}}(n(e))$, where $\mathcal{F}=\{(a, b) \in$ $\left.E, a \in V_{i} \wedge b \in V_{j} \wedge i \neq j\right\}$ subject to the balance constraint $W_{i} \leq W_{\text {avg }}(1+$ $\epsilon)$ for $i=1, \ldots, k$. In the above formula, $W_{i}$ is the sum of the weights of all vertices in part $V_{i}$ and $W_{\text {avg }}=\sum_{u \in V} n(u) / k$ represents the weight of each part in $G$ under the perfect load balance. Moreover $\epsilon$ denotes the maximum imbalance tolerance as a percentage of the ideal weight where typical values are between $2 \%, 5 \%$, or $10 \%$. The total edgecut is a classic metric, known to approximate the total communication volume of a parallel application [17] and is used here as a representative of the partitioning quality.

\subsection{Multilevel Framework}

Despite the computational complexity of the graph partitioning problem (NP-complete [18), many heuristic algorithms have been proposed in the past 
that find reasonably good partitions. Such examples are combinatorial methods that explore local or global solutions working on the graph structure or spectral

$(O(k))$. Nevertheless the result of the initial partitioning phase can be crucial for the final quality of the projected solution back to $G_{0}$.

Finally, during the uncoarsening phase each vertex of the finer graph is assigned to the same part as its counterpart vertex in the coarser graph. However 


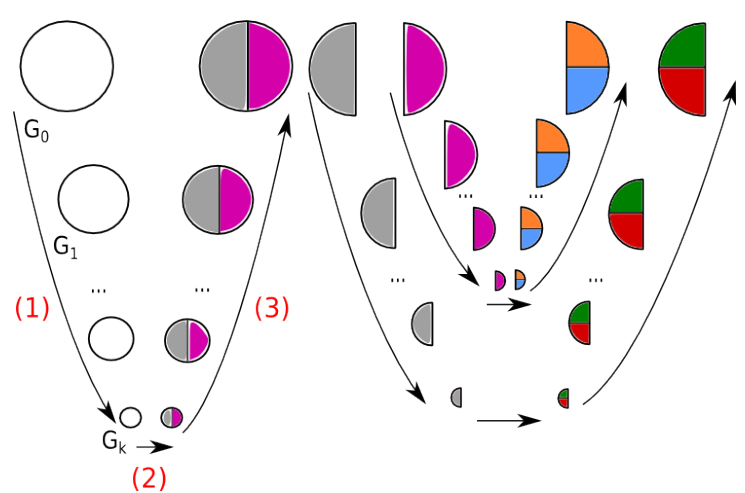

(a) MLRB (W cycle)

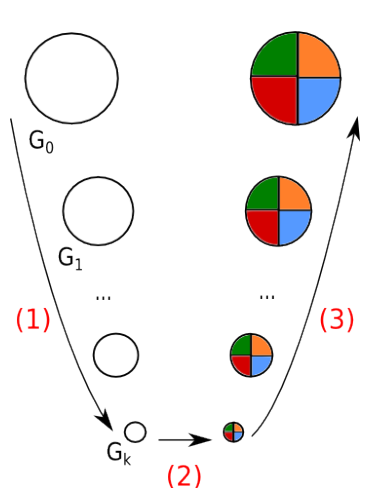

(b) MLKW (V-cycle)

Figure 2: Illustration of a 4-way partitioning with MLRB and MLKW algorithms : (1) coarsening, (2) initial partitioning and (3) uncoarsening phases of the multilevel frameworks. 
In a V-cycle, the multilevel framework is called just once. and the coarsest graph is now directly partitioned into $k$ parts. Note that refining a $k$-way partitioning is considerably more complicated than refining a bisection, so the uncoarsening

225 phase of a MLKW algorithm may be more time consuming [21]. Indeed, the time complexity of MLKW is dominated by the complexity of the uncoarsening step which is $O(k|E|)$ in the general case. However clever algorithms that achieve a complexity of $O(|E|)$ exist, such as the one proposed in [21].

\subsection{Tools for Partitioning with Fixed Vertices}

In Table 1, we present some useful information about common graph and hypergraph partitioning tools, such as ScOtch, Metis or PaToH. As one may see, more than half of the given tools do not handle at all problems with initially fixed vertices despite the research interest on this problem. Note that this is especially true for graph partitioning tools. Moreover, among the partitioning tools that solve this problem, the majority uses the RB method combined with the multilevel framework (MLRB or MLKW with RB). As we mentioned in Section 1. even though RB is very efficient for the classic graph partitioning problem, it has limitations when fixed vertices incur in the partitioning. However, some tools propose alternative solutions for the above problem and we review them here in detail. We also review RB.

An algorithm that addresses graph problems which involve initially fixed vertices is the one proposed in RM-Metis [6]. RM-Metis is used to solve the $k$-way repartitioning problem and thus assumes an existing partition that has become imbalanced. As mentioned before, in such re-balancing problems at least one fixed vertex per part is included in the graph in order to model more efficiently the load re-distribution. The main idea of the algorithm is to apply greedy growing techniques, selecting $k-1$ growing parts and a shrinking one, until the load of every part drops below the maximum allowed part size. As a 
TABLE 1: Graph and hypergraph partitioning tools.

\begin{tabular}{|l|cccccc|}
\hline Tools & Type & Fixed & Parallel & Scheme & Initial Part. & Available \\
\hline METIS 24] & graph & no & no & MLRB & - & source \\
KMETI [21] & graph & no & no & MLKW & RB & source \\
ParMetis 24] & graph & no & yes & MLKW & RB & source \\
Scotch 25] & graph & yes & no & MLKW & RB & source \\
PT-Scotch 25] & graph & no & yes & MLRB & - & source \\
RM-Metis [6] & graph & only $k$ & no & MLKW & greedy & no \\
KaHIP [26] & graph & no & yes & MLKW & RB & source \\
Chaco 20] & graph & no & no & MLRB & spectral & source \\
KGGGP [1] & graph & yes & no & MLKW & greedy & source \\
HMetis 24] & hypergraph & yes & no & MLRB & - & binary \\
KHMetis 24] & hypergraph & no & no & MLKW & RB & binary \\
PAToH [27] & hypergraph & yes & no & MLRB & - & binary \\
KPAToH [16] & hypergraph & yes & no & MLKW & RBBGM & no* \\
ZoLTAN (PHG) 28] & hypergraph & yes & yes & MLRB & - & source \\
Mondriaan [29] & hypergraph & no & no & MLRB & - & source \\
\hline
\end{tabular}

result the problem resumes in selecting a good shrinking part. A limitation of RM-METIS is that it is designed only for repartitioning and that it may results in a shrinking part of inferior quality (not smooth frontiers). Finally this algorithm is implemented inside the multilevel framework of METIs, but unfortunately its implementation is not publicly available. For the above reasons we choose not to focus on RM-Metis in this study.

Following, we describe in detail the three methods that are part of our experimental study. More precisely we first discuss the RB methodology particularly for problems with fixed vertices (Section 3), then we review the RBBGM algorithm that was first introduced in $\mathrm{KPATOH}$ (Section 4) and finally, we present the KGGGP method (Section 5).

\section{Recursive Bisection for Fixed Vertices (RB)}

Classic RB based methods follow a simple divide \& conquer strategy : the original graph is first split in two parts (bisection) and this procedure is recursively repeated independently on the two resulting subgraphs, until the desired number of parts is obtained. Thus, it is possible to represent this procedure with a bisection tree (Figure 3) which illustrates the implicit part numbering scheme used by RB. 
Let us now assume that the number of desired parts at the end of the partitioning is $k$. Note that at each bisection step, RB selects half of the available part numbers and assigns them to one of the two resulting subgraphs. If this step were to be performed in an exhaustive way, RB would have to try $k$ ! combinations before choosing the best solution. In other words, there are combinatorially many part numbering selections for each bisection step. Therefore, to avoid choosing among $k$ ! combinations, $\mathrm{RB}$ decides to blindly group together parts with consecutive part indices, following an inherent rule. For instance in the first bisection, RB will try to assign the first half of all the part indices, $[1, k / 2]$, to one subgraph and the second half, $[k / 2+1, k]$, to the other. At each level of the bisection tree, the same methodology is applied.

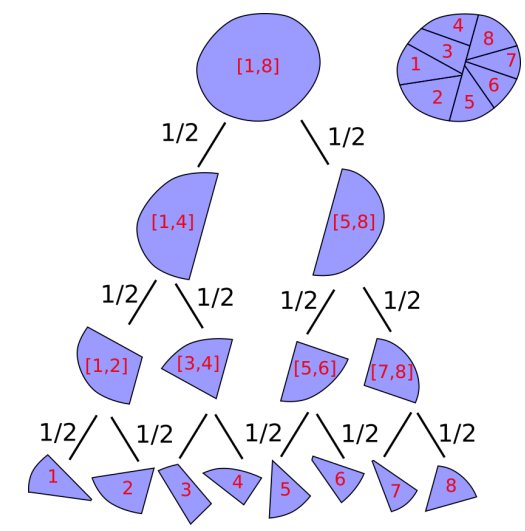

Figure 3: Illustration of the RB method : bisection tree and its implicit part numbering (in red) for a 8-way partition.

Moreover, RB can be extended in a straightforward way to include problems with initially fixed vertices. Again, during the first bisection, fixed vertices that are originally pre-assigned to part indices $[1, k / 2]$ are fixed to part 1 while fixed vertices that are assigned to parts $[k / 2+1, k]$ are considered as fixed to part 2 . This idea is applied recursively in every bisection.

Now let us reconsider the example presented in Section 1 (cf 1.1) where a graph with a "bad" fixed vertex scheme is partitioned in four parts. If we 

experiments that confirm our observations follow in Section 6

\section{Recursive Bisection with Bipartite Graph Matching (RBBGM)}

An algorithm for hypergraph partitioning is introduced in [16] that successfully handles problems with initially fixed vertices. In this work, the authors

examine the result of $\mathrm{RB}$, we clearly see that the algorithm cannot select a good bisection between parts $[1,2]$ and $[3,4]$ that simultaneously respects the constraint of initial fixed vertices during the first bisection. As a result, the final partitioning quality is considerably poor for the RB based method.

To better understand what goes wrong in this case, let us consider the Figure 4 that reproduces the same experiment on a smaller $10 \times 10$ grid graph. Here, we realize the same 4-way partitioning under the constraint of fixed vertices depicted in Figure 4a. Following a similar methodology as Simon and Teng [30, one may clearly see that RB does not succeed in finding the optimal solution under the constraint of fixed vertices. This is because RB tries at each step to find the optimal local solution, and in this case the first optimal bisection involves disconnected components as shown in Figure 4b (i.e. vertices fixed to part three are disconnected from the part containing vertices fixed to part four and the same happens for vertices fixed to parts two and one). Note that $\mathrm{RB}$ cannot find a better bisection that puts fixed vertices of parts one and two in a single connected component. Another possible solution for the first bisection would give edgecut of higher cost (not optimal). Therefore, the first "bad" bisection of RB is maintained in the final solution, which is clearly not optimal : the best solution of RB is presented in Figure $4 \mathrm{c}$ compared to the optimal solution shown in Figure 4d. The above examples are just an illustration of the problematic behavior of RB methods with initial fixed vertices. Further

\footnotetext{
identify the inferior performance of $\mathrm{RB}$ when fixed vertices are involved in the
} 


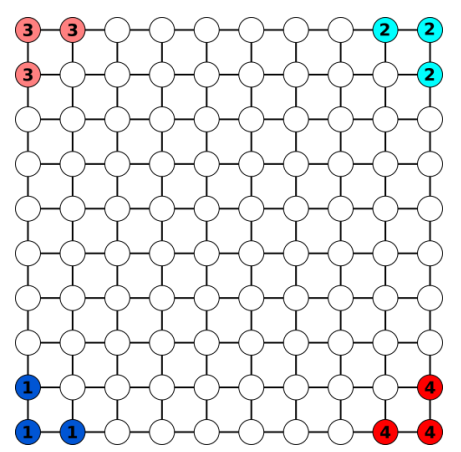

(a) Initial fixed vertices.

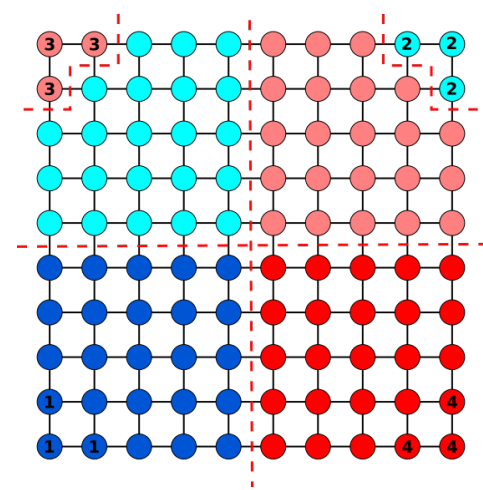

(c) Best solution for RB.

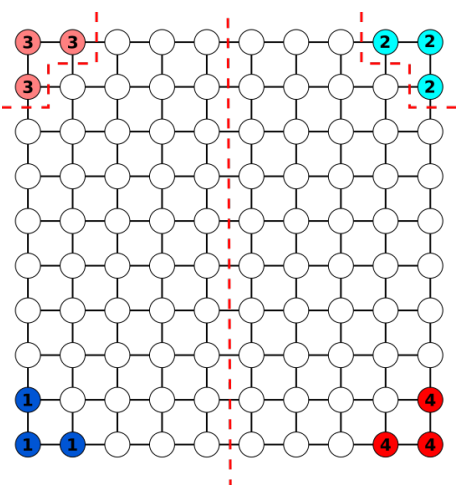

(b) Optimal first bisection.

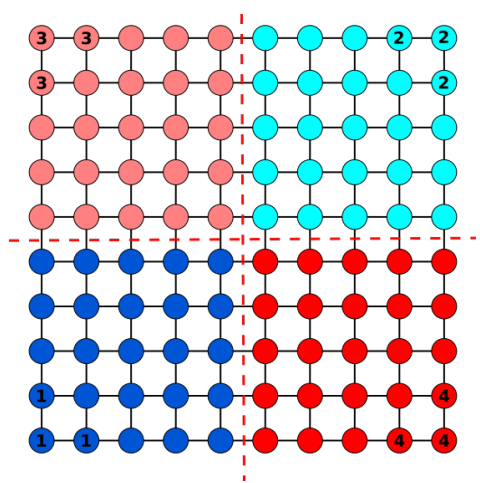

(d) Optimal solution.

FIGURE 4: Issues of the RB method under the constraint of initial fixed vertices. While finding the optimal first bisection, the best solution of RB to the 4-way partitioning problem will not be optimal, showing an additional cost of 8 edges cut.

process, mentioning its inability to explore the combinatorially many part labelings that correspond to a given fixed vertex configuration. To correct the above deficiency, they propose a new multilevel direct $k$-way hypergraph partitioning that uses a RB-based algorithm and an additional post-processing technique to relabel the resulting parts such that the edgecut remains minimized. They refer to the above algorithm as RBBGM and they provide an implementation called KPAToH based on modifications of the multilevel framework of PATOH.

For the coarsening phase of KPAToH, a modified Heavy Connectivity Mat- 
ching 1 is proposed such that no two fixed vertices are matched together at any coarsening level. However, a fixed vertex can be matched with any free vertex, forming a fixed super-vertex for the next level. Therefore, the number of fixed super-vertices in the coarsest level is equal to the number of initially fixed vertices of the hypergraph. As always, free vertices are matched together according to the chosen heuristic.

325

During the initial partitioning phase of KPAToH, fixed vertices are temporarily removed from the coarsest hypergraph and a partitioning of the resulting hypergraph is performed with a classic RB-based algorithm. Once the partition is computed, fixed vertices are re-introduced in the hypergraph according to a relabeling strategy. Note that if no relabeling is used, fixed vertices are simply re-assigned to the parts based on their initial part numbering. In this case, the final partition may not be optimized in terms of net cost minimization, since nets incident to fixed vertices are not considered during RB. In other words, a relabeling strategy that minimizes the net cost contribution of re-introduced fixed vertices is necessary to obtain an optimized partition for the coarsest hypergraph.

The problem of relabeling fixed vertices is formulated in $\mathrm{KPATOH}$ as a maximum weighted bipartite graph matching problem, that represents the minimum increase of edgecut. In the proposed formulation, sets of fixed vertices and resulting parts form the two node sets of the bipartite graph $B=(X, Y)$. More precisely, each vertex $X_{i}$ in $B$ represents fixed vertices, initially assigned to part $i$, while each vertex $Y_{i}$ represents vertices that belong to part $i$ after the partitioning. Additionally, the bipartite graph contains all possible edges $\left(X_{i}, Y_{j}\right)$ between fixed vertices and ordinary ones with a weight that corresponds to the sum of weights for edges with incident vertices in both $X_{i}$ and $Y_{j}$.

1. HCM is the equivalent algorithm of HEM for hypergraph partitioning 


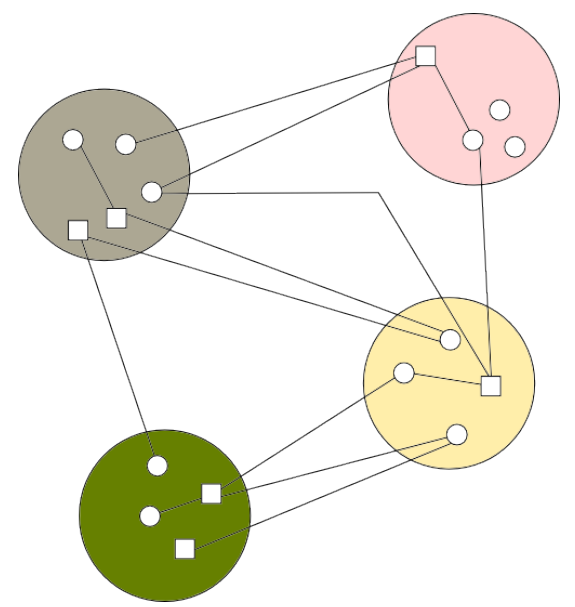

FiguRE 5: Example of coarsest graph where each color corresponds to a different part. Fixed vertices are represented as squares and free ones as circles.

Following, we give an example of the initial partition phase of $\mathrm{KATTOH}$ on a graph structure rather than on a hypergraph for reasons of consistency with the focus of this work. Figure 5 shows the partitioning result of a graph with fixed vertices using KPATOH and no relabeling strategy is used to reassign fixed vertices. That is, the modified graph which contains only free vertices has been partitioned in four parts with RB while fixed vertices have been simply re-introduced in the graph after the partitioning, maintaining their initial part assignment. Note that fixed vertices are represented in the figure as squares while free vertices as circles. Additionally, for ease of presentation, unit edge weights are assumed and only edges connecting fixed vertices and free ones are displayed, since any additional edgecut contribution may be due to such edges.

In this partitioning example, the upper bound of edgecut contribution after fixed vertices are re-introduced in the graph is 14 (edges). In Figure 6, we present two instances of the bipartite graph that models the re-assignment of fixed vertices for this example, one (6a) that corresponds to no relabeling and a second one $6 \mathrm{~b}$ which follows a relabeling strategy. Finally, note that sets of 
fixed and (previously) free vertices that are assigned to the same part are drawn in both figures with the same color. Therefore, in the case of no relabeling, the edgecut increase is 10 and implies an edgecut saving of four (colored edges). However, it is easy to see that there is an assignment of fixed vertices that leads to higher egdecut saving and is the solution of the maximum-weight bipartite graph, illustrated in 6b. This relabeling obtains the highest saving of edgecut which leads to the minimum cost increase of $14-7=7$, instead of 10 .

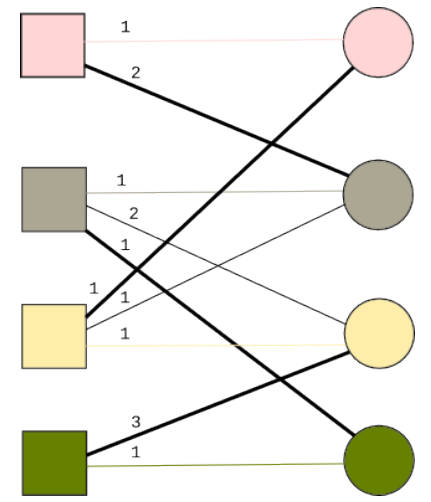

(a) No relabeling.

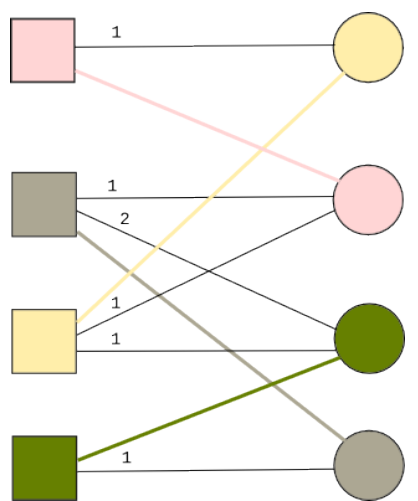

(b) Relabeling strategy of minimized cost contribution.

FIGURE 6: The bipartite graph used for the reassignment of fixed vertices in the example shown in Figure 5 A square vertex represents a set of fixed vertices $X_{i}$, while a circle vertex represents a part $i$

For the uncoarsening phase, a modified version of the $k$-FM refinement algorithm is used where fixed vertices are locked to their respective parts and are not allowed to move between parts.

Experiments on hypergraphs with fixed vertices performed by $\mathrm{kPATOH}$ show a net cost improvement of overall average between $17 \%$ and $21 \%$ compared to the multilevel RB-based method used in PAToH. Finally note that RBBGM has an important limitation as it does not handle the imbalance issues that may incur if fixed vertices are not evenly distributed to parts.

Below we discuss our implementation of RBBGM for graphs. 


\section{Discussion on Graph Implementation of RBBGM}

We choose to implement RBBGM inside the multilevel framework of SCOTCH for two main reasons. First, SсотCH is one of the most widely used graph par-

380 titioning tools, with a fast implementation of the multilevel framework and an easy programming interface. But more importantly, $\mathrm{SCOTCH}$ already includes a RB-based method that addresses the problem of graph partitioning with initially fixed vertices that can be used as a reference method. Remember that the multilevel framework consists of multiple heuristics and each partitioning tool has different parameters for the coarsening, initial partitioning and uncoarsening phase, that may heavily influence the quality or execution time of the final solution. Therefore, in order to conduct a reliable comparison of different methods used for the initial partitioning phase (such as RBBGM and KGGGP), one should implement them all inside the same multilevel framework. Finally, note that in order to solve the maximum weighted bipartite graph matching problem that appears in RBBGM, we use an implementation of the Hungurian algorithm [31] which finds an exact solution and has a complexity of $O\left(k^{3}\right)$.

\section{The KGGGP Algorithm}

In this section, we describe a direct $k$-way graph partitioning algorithm, called KGGGP ( $k$-way greedy graph growing partitioning), which can be easily integrated in a multilevel framework and successfully handles any number of initially fixed vertices. The algorithm has been initially proposed in [1] where a detailed description is provided. However in order to keep this work selfcontained we dedicate here a substantial discuss on KGGGP.

The KGGGP algorithm is an extension of the standard greedy bipartitioning [32, 33] where vertices are iteratively added into two growing parts according to a minimization criterion. KGGGP uses the same idea for a direct $k$-way 
partitioning where $k$ parts (instead of just two) are growing simultaneously, in a concurrent procedure. In a certain way, KGGGP can be seen as a variation of which initially contains all free vertices and becomes empty at the end of the procedure.

\subsection{Algorithmic description}

Let us consider a graph $G=(V, E)$ and an initial partition $P$, such that a loop, the KGGGP algorithm selects the best global displacement $(v, p)$, that is, the one having the maximum gain, subject first to the balance constraint $(\mathcal{B})$ and secondly to the connectivity constraint $(\mathcal{C})$. While the first constraint enforces the development of balanced parts, the second constraint ensures that each part 425 a vertex $v$ in the neighborhood of the growing part $p$, as far as possible. Once a valid displacement $(v, p)$ is chosen, $v$ moves to the target part $p$ (i.e, $P[v] \leftarrow p$ ) and all displacements $(v, \star)$ to other parts are removed from consideration. At this point, we need to update the gain of all displacements $\left(v^{\prime}, \star\right)$, that involves 

no more free vertices in $P$.

Besides, KGGGP uses a similar data structure as in FM adapted here for $k$ parts. This structure is called gain bucket and allows a quick locate of the best global displacement. In practice, we use three instances of the gain bucket structure to store and select displacements efficiently : one regular bucket and two additional buckets to manage the displacements that do not respect the connectivity or the balance constraint. In Figure 7, we illustrate a diagram of all possible moves of a displacement between the three buckets during the selection phase. Each transition may happen exactly once for each displacement.

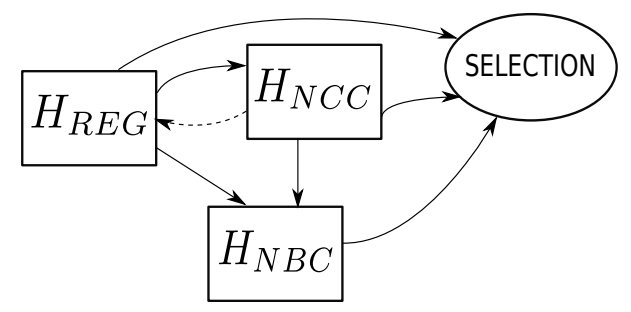

Figure 7: Diagram of all possible moves of a displacement during the selection phase. $H_{R E G}$ corresponds to the regular gain bucket, while $H_{R E G}$ and $H_{R E G}$ correspond to the connectivity and balance gain buckets respectively.

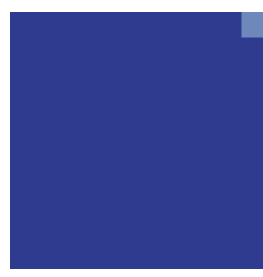

(a) Step 100

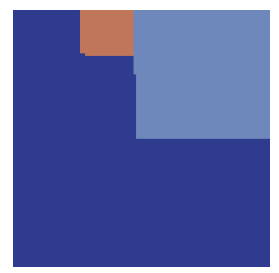

(b) Step 3000.

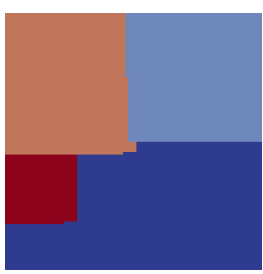

(c) Step 6000 .

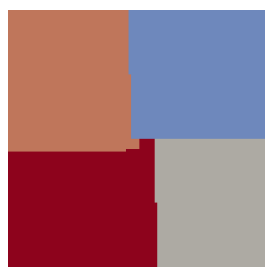

(d) Step 10000.

Figure 8: Steps of KGGGP while partitioning a $100 \times 100$ grid graph in 4 parts. The free part is colored in blue. This part becomes empty at the final step (10000) showing a 4-way partition.

In Figure 8, we illustrate the evolution of the part growing when a simple $100 \times 100$ grid graph is partitioned into 4 parts using the KGGGP algorithm without a multilevel framework. As one may see, the algorithm aims to respect 
both the balance and the connectivity constraints leading to a rather balanced and connected partition even with no refinements.

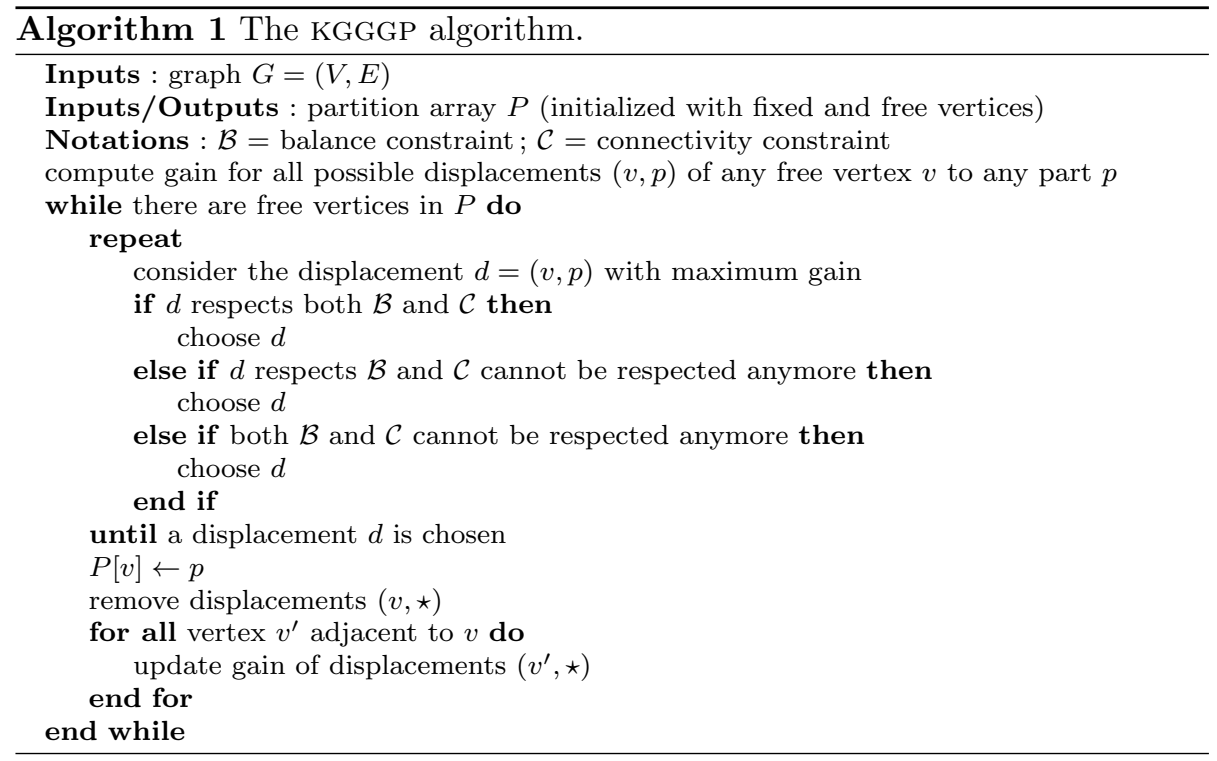

\subsection{Fixed Vertex Management}

Note that it is very straightforward to handle initial fixed vertices within KGGGP. More precisely, fixed vertices are directly placed in their respective parts before the algorithm starts and are simply not considered as candidates for any displacement. Obviously, these vertices are not ignored : they influence the balance among parts and are taken into account in the gain calculation of free vertices in the neighborhood.

\subsection{KGGGP in a Multilevel Framework}

The KGGGP algorithm can be easily integrated in any multilevel framework with some simple adjustments regarding the initial fixed vertices. Firstly, during the coarsening phase, an extra constraint is added, so that fixed vertices which belong to different parts can not be matched together, while they may 
be matched with free vertices. Following, we partition the coarsest graph with KGGGP as it is described above and we continue with the uncoarsening phase, where refinements for $k$-way partitioning are performed to further improve the final result. Note that during this phase, we maintain all fixed vertices locked, forcing them to remain in place.

\subsection{Gain Formulas for Minimization Criterion}

As we mentioned above, there exist multiple minimization criteria to determine displacement selection. Here, we present three of them : the classic gain minimization as it is presented in FM algorithm, the diff gain proposed by Battiti and Bertossi 33 and finally a hybrid minimization criterion.

Assuming that a vertex $v$ moves into part $p$, we divide its incident edges $\left(v, v^{\prime}\right)$ in three categories : the internal edges such that $\operatorname{part}\left[v^{\prime}\right]=p$, the external edges such that $\operatorname{part}\left[v^{\prime}\right] \neq p$, and the free edges such that $\operatorname{part}\left[v^{\prime}\right]=-1$. Let $N_{\text {int }}(v)$ be the number of internal edges, $N_{\text {ext }}(v)$ the number of external edges and $N_{\text {free }}(v)$ the number of free edges, for $v$. Clearly, $N_{\text {int }}(v)$ measures how strongly $v$ is connected to the part $p$, while $N_{e x t}(v)$ measures how strongly it is attracted to other parts (except -1$)$. The parameter $\alpha$ is an integer constant that is used to enforce the part connectivity once again ; more precisely, it favors internal edges compared to other edges. Typical values of $\alpha$ are in the range 1 to 10 .

TABLE 2: Description of minimization criteria.

\begin{tabular}{||l|l||}
\hline Criterion & Gain Formula \\
\hline classic & $G=\alpha \cdot N_{\text {int }}(v)-N_{\text {free }}(v)$ \\
diff & $G=\alpha \cdot N_{\text {int }}(v)-N_{\text {ext }}(v)$ \\
hybrid & $G=\alpha \cdot N_{\text {int }}(v)-N_{\text {ext }}(v)-N_{\text {free }}(v)$ \\
\hline
\end{tabular}

Based on the above definitions, the Table 2 presents the gain formulas of the different criteria. The main difference between those formulas is due to the free edges, depending on whether they are considered as external or not. 
The main steps of the KGGGP algorithm consist of initializing displacements, selecting displacements and updating the bucket structures after a displacement is performed. The initialization step computes the gain for $k \times|V|$ possible displacements in $O(k|E|)$, as the gain calculation depends on the neighborhood of each vertex. Then, KGGGP selects iteratively $|V|$ displacements and updates the buckets for each displacement in $O(k|E|)$, since it visits the neighborhood of each selected vertex for all parts. Therefore, the total time complexity of KGGGP is $O(k|E|)$. As a reminder, the time complexity of $\mathrm{RB}$ is $O(\log (k)|E|)$. Finally, the memory complexity is mainly due to saving all possible displacements in the gain bucket data structure, which is $O(k|V|)$.

\subsection{Optimization : Local Greedy Approach}

In order to reduce the total time complexity of KGGGP, we implement a second version of the method, where we enforce local selection of displacements instead of the global selection described above. The key idea here is to search for upcoming displacements only in the neighborhood of vertices that belong already to a part. This approach is similar to the one used in KMETIS to optimize the $k$-way FM refinement heuristic 21].

As a result, we do not need in the initialization step to compute a priori the gain value for all possible displacements. Instead, after a displacement $(v, p)$ is chosen, we dynamically insert in buckets new displacements $\left(v^{\prime}, p\right)$ for all remaining free vertices $v^{\prime}$ in the neighborhood of $v$. If there are no more displacements available in buckets while the partition is not complete, the method switches back to the global approach for the remaining free vertices, giving a time complexity of $O(k|E|)$ in the worst case and $O(|E|)$ in the best case. 


\section{Experiments}

In this section, we present the experimental results on the partitioning quality and time performance of different algorithms. For our experiments, we implement two versions of the KGGGP algorithm, one that follows the global greedy approach (KGGGP_G) and one that follows the local approach (KGGGP_L) as described in Section 5. Following, we compare the above methods with our RBBGM implementation of $\mathrm{KPATOH}$ (explained in Section 4) and the default RB-based method of Scotch (explained in Section 3. Both codes of KGGGP and the one of RBBGM are publicly available in the MetaPart library at http://metapart.gforge.inria.fr.

All algorithms are implemented inside the same multilevel framework (that of SCOTCH v6.0), as part of the initial partitioning phase of a MLKW method. Moreover, they are tuned with exactly the same partitioning parameters : imbalance tolerance of 5\%, HEM for coarsening, maximum coarsest graph size equal to $30 \times k$, FM refinement with 10 passes, and a maximum number of negative moves allowed set to 100 for each refinement pass. As a result, the above configuration allows us to fairly compare the impact of different algorithms on the final partitioning solution. In the remainder of this section, we will refer to the default RB method of SCOTCH as "SCOTCH", and it will serve as a reference for the relative comparisons.

In this study, we perform three different experiments. First, we present a preliminary experiment that evaluates the best internal parameters for KGGGP_G and KGGGP_L and then we perform two experiments that test our methods on graph instances with initially fixed vertices. Note that a previous study including hypergraph partitioning tools (ZOLTAN and PATOH) has been presented in [1] and provides an insight on the general performance of those tools under the fixed vertex paradigm. Here, we extend the above comparison on a larger collection of 
graphs but we restrict our experiments only on partitioning methods for graph structures. Finally, experiments were conducted on a machine with two fourcore Intel Xeon CPUs, running at $2.6 \mathrm{GHz}$ with $24 \mathrm{~GB}$ memory. All algorithms ${ }_{535}$ are implemented in $\mathrm{C}$ and are compiled in $G C C$ with -O3 optimization flag.

The dataset used in this study represent real-life applications from different scientific domains (numerical simulations, clustering problems and road networking) available in the public DIMACS'10 collection [34. One may find in Table 5 the different graph categories that are used here along with some useful infor540 mation, such as the total number of vertices or edges, for each graph. Besides, each experiment is performed 5 times for every graph using randomly generated seeds each time.

To thoroughly analyse our results, we include here two different types of figure. In Figures 11 and 13 , for each graph category, we present normalized re545 sults relative to $\mathrm{ScOTCH}$ on the average values over all included graphs (within that category). In these figures, the $\mathrm{x}$-axis represents the edgecut and the $\mathrm{y}$-axis represents the execution time, while the number of desired parts increases from 10 to 500. Moreover, to analyze our experiments in a global perspective, we include figures that demonstrate average values of the obtained results (either the 550 edgecut or the execution time) over the entire graph collection as the number of parts increases (Figures 9 910 12). Note that the error bars in those latter figures indicate the standard deviation for each method. Finally, whenever a method fails to respect the balance constraint, its results are not taken into account in the average calculations. Obviously by doing so, we may favor methods that fail 555 to compute a valid partition. To address this problem, we carefully examine the success rate of each partitioning tool in addition to the main metrics. 


\subsection{Tuning of KGGGP without Multilevel Framework}

The goal of this preliminary experiment is to tune some important parameters of the KGGGP method. More precisely, we want to evaluate the best KGGGP_L outperforms KGGGP_G for runtime performance.

\subsection{Experiments with Fixed Vertices}

We present results with fixed vertices from two experimental cases, each one representing a different way to distribute initially fixed vertices to the input graph. We believe that the two proposed experiments represent configurations of fixed vertices that may appear in real life problems in areas such as circuit design or dynamic load balancing. We denote these schemes bubble and repart. Moreover, in these experiments, we decide to include only the local version of 


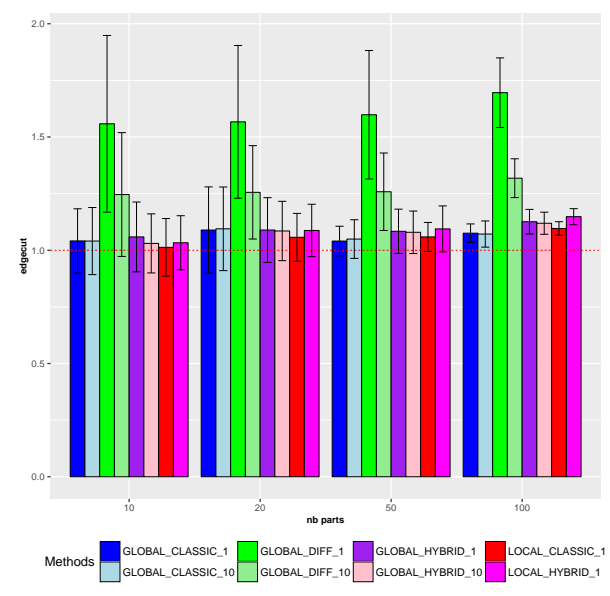

(a) Edgecut.

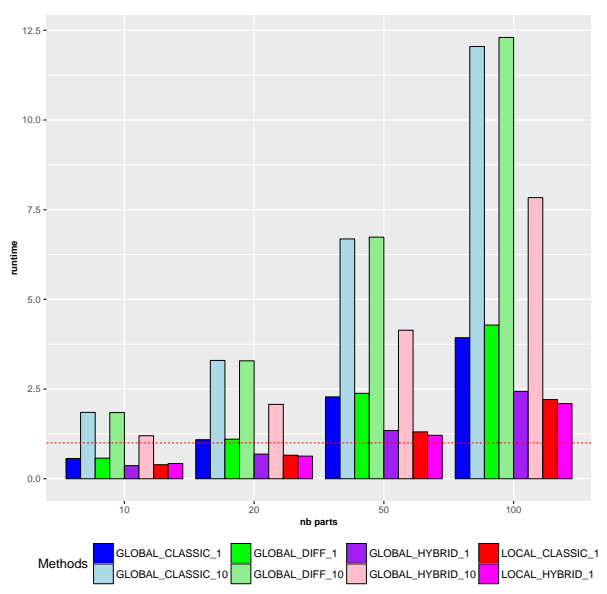

(b) Runtime.

Figure 9: KGGGP evaluation on the Walshaw collection relatively to SCOTCH, without multilevel framework. Comparison of the three gain formulas (classic, diff and hybrid) for a parameter $\alpha$ equal to 1 or 10, and comparison of the global and local version of KGGGP.

KGGGP (KGGGP_L) and not the global one. Indeed, based on the results of the previous experiment, KGGGP_L is clearly faster than KGGGP_G while it produces partitions with similar quality.

\subsubsection{Bubble Scheme}

The idea behind the bubble scheme is to create an initial graph structure that already has a number of fixed vertex subsets (bubbles). To make the scheme more generic, we assume that these subsets do not have the same size. We believe that this scheme may represent configurations that come from two-step partitioning methods as explained in Section 1 under the context of multi-physics simulations. To create the bubbles, we compute $k$ seeds that are as distant as possible from each other (based on a BFS [35. technique) and we fix one seed per part randomly. Each seed is later used as the center of a fixed vertex bubble.

In particular, each bubble grows using BFS such that neighboring vertices are assigned to the same part as the center of the bubble. The procedure continues 
until each bubble reaches a certain percentage of ideal size (assuming vertex weights of 1). In this scheme, we allow different size among the bubbles that

results of this experiment, in Figure 10b we see that both KGGGP_L and RBBGM perform, on average, better than SCOTCH until the number of parts reaches 200 ( $7 \%$ faster for KGGGP_L and $2 \%$ for RBBGM), but become (21\%) slower than ScOTCH when the number of parts is 500 . to SCOTCH. For KGGGP_L, this is not a surprise considering its theoretical complexity that depends on the number of parts. Additionally, it is important to note that if a heuristic solution were used instead of the Hungarian algorithm, the time performance of RBBGM might have been optimized. However, in this study we favor an exact solution to the maximum weighted bipartite graph problem, since the performance of this step is not of critical importance.

Moreover in Figure 11, we present the same results but we depict each graph category separately. This representation allow us to evaluate each method upon different graph structures. Additionally, to facilitate the experimental descrip620 tion we group the graph collections in two different categories, namely group 1 and group2. More precisely, group1 includes either graphs that derive from numerical simulations (Matrix, Numerical and Dynframe) or graphs that are deliberately chosen for partitioning (Walshaw). On the other hand group2 includes graphs from the Clustering and Streets collections that appear to be more dif- 


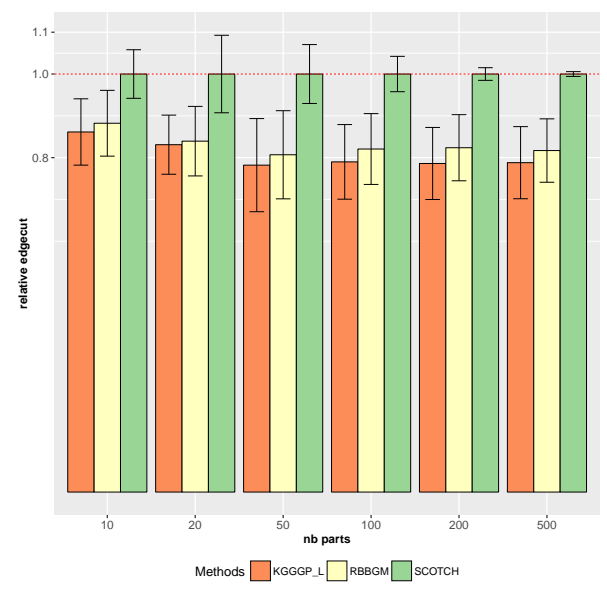

(a) Edgecut.

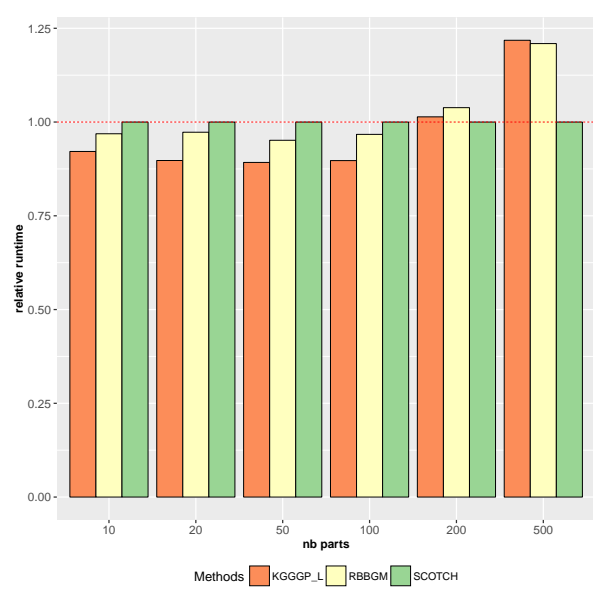

(b) Runtime.

Figure 10: Average results over the entire DIMACS'10 collection (bubble scheme).

Additionally, if we focus on the performance results of group2, we see that KGGGP_L is on average $27 \%$ faster that Scotch and $24 \%$ than RBBGm. This leads us to believe that a $k$-way graph partitioning may be more suitable for such graph structures. On the other hand, if we examine the performance results of group1 we confirm the scalability issues of KGGGP_L but we also remark that the poor performance of the global results (in Figure 10b comes mainly from the Walshaw collection when the number of parts is 500. Indeed in this case KGGGP_L is 2.5 times slower than Scotch.

Finally, note that both SсотCH and RBвGM have difficulties finding a parti- 


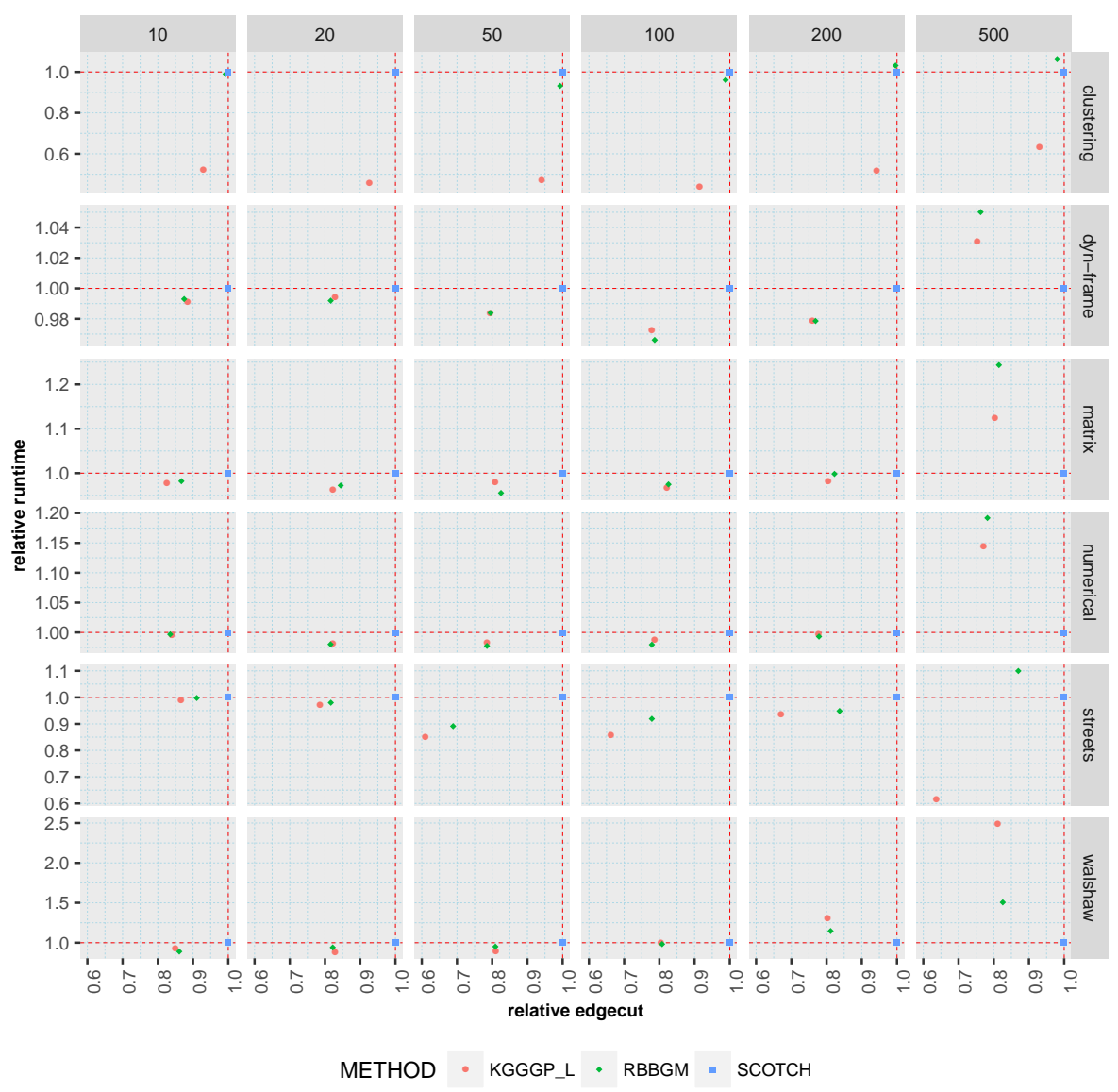

Figure 11: Average results on the edgecut quality and time execution for each group in the DIMACS'10 collection (bubble scheme).

tioning solution that respects the imbalance tolerance and often exhibit a large number of failures. In Table 3 , we demonstrate the average percentage of failures for each method over all number of parts, depicted for each collection. In this context, we may say that KGGGP_L is more robust since it fails only for the ${ }_{645}$ Walshaw collection when the number of parts is 500 . 
TABLE 3: Average failure percentages for each method per graph collection over all number of parts (bubble scheme).

\begin{tabular}{|rrrrrrr|}
\hline method & streets & matrix & numerical & clustering & dynframe & walshaw \\
\hline SCOTCH & $42 \%$ & $1 \%$ & $10 \%$ & $13 \%$ & $3 \%$ & $10 \%$ \\
RBBGM & $48 \%$ & $3 \%$ & $15 \%$ & $12 \%$ & $1 \%$ & $10 \%$ \\
KGGGP_L & $0 \%$ & $0 \%$ & $0 \%$ & $0 \%$ & $0 \%$ & $1 \%$ \\
\hline
\end{tabular}

\subsubsection{Repart Scheme}

For the repart scheme, we follow the repartitioning method proposed by ZOLTAN in [7] where an initially balanced partition becomes deliberately imbalanced. Here, the initial partition is obtained with a RB-based method and the size of each part is linearly increased from $0 \%$ to $50 \%$, by changing the weight of random vertices in each part. It is important to mention that the part numbering of the initial partition is respected in the imbalanced result. Following, we build an enriched graph of the resulting partition, adding a single fixed vertex per part along with migration edges that connect the fixed vertex with its respective part. In order to enforce the connectivity of fixed vertices with their respective parts, we add a relatively important weight to the migration edges. Note that the enriched graphs are larger in size compared to the original ones, thus we test our algorithms on a sub-collection of the graph set in Table 5 omitting the large collections Dynframe and Streets.

For this experiment, global results on the edgecut and partitioning time are presented in Figure 12 while the same results are also depicted separately for each group of graphs in Figure 13. In Figure 12a, we see that KGGGP_L obtains minimized edgecut results for the entire collection with an average gain of $11 \%$ compared to Scotch and $18 \%$ compared to RBBGM. Moreover, as seen in Figure 13, KGGGP_L may reach an edgecut minimization of up to $30 \%$ compared to Scotch and up to $36 \%$ compared to RBBGM for some collections (for instance, the Numerical one). Additionally, we observe that RBBGM does not globally produce partitions of high quality for this experiment and thus may 
not be a suitable solution for a scheme such as repart. A possible explanation is that the maximum-weight graph matching problem that should be solved here is more complicated due to the large number of migration edges. As a result the additional edgecut of re-introducing fixed vertices to the graph is rather significant. A second explanation is that RBBGM removes the fixed vertices before repartitioning and thus loses the ability to minimizing the data migration between the initially imbalanced input and the final balanced partition.

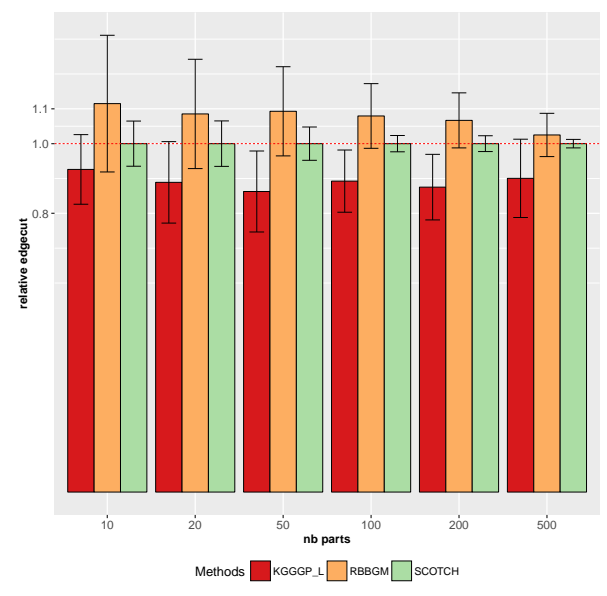

(a) Edgecut.

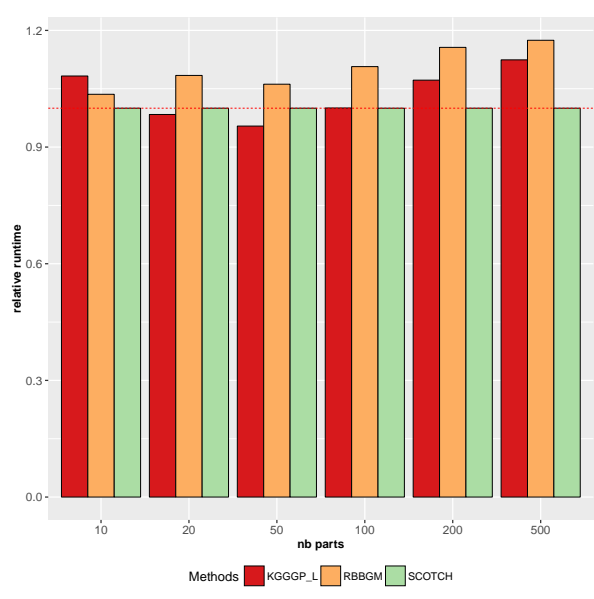

(b) Runtime.

Figure 12: Average results over the entire DIMACS'10 collection (repart scheme).

Regarding the runtime performance of this experiment, in Figure $12 \mathrm{~b}$ one may see that SCOTCH is on average the fastest method followed by KGGGP_L with an average performance overhead of $2 \%$ while RBBGM is the slowest method with $9 \%$ overhead compared to SCOTCH. Even though KGGGP is not much slower than SсотCH, these results confirm again the poor scalability of KGGGP_L as the number of parts increases. However as one may see in Figure 13 for certain group of graphs (Numerical and Matrix), KGGGP_L is faster than ScOTCH even when the number of parts increases. Finally, the average percentage of failures over the number of parts for this experiment can be found in Table 4 . 
TABLE 4: Average failure percentages for each method per graph collection over all number of parts (repart scheme).

\begin{tabular}{|rrrrr|}
\hline method & matrix & numerical & clustering & walshaw \\
\hline SCOTCH & $13 \%$ & $4 \%$ & $7 \%$ & $15 \%$ \\
RBBGM & $0 \%$ & $0 \%$ & $0 \%$ & $4 \%$ \\
KGGGP_L & $0 \%$ & $0 \%$ & $0 \%$ & $0 \%$ \\
\hline
\end{tabular}

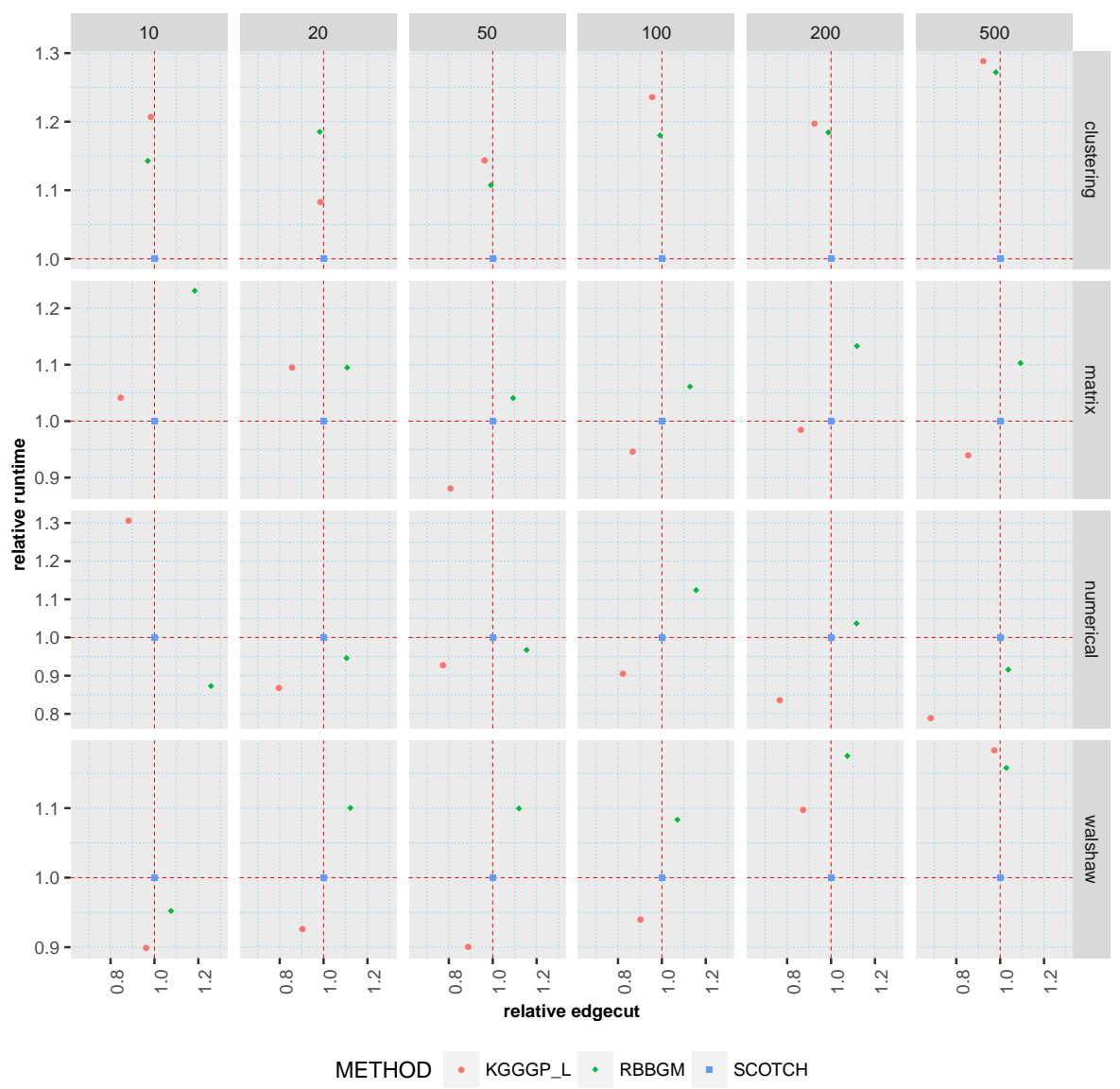

FIGURE 13: Average results on the edgecut quality and time execution for each group in the DIMACS'10 collection (repart scheme).

\section{Conclusion}

This work is driven from our interest in graph partitioning with initially fixed vertices that appear in many scientific problems, such as the dynamic load 
balancing of large-scale applications. Under this model, additional constraints of the underlying problem are represented in the graph with initially fixed vertices. Unfortunately, in such settings the state-of-the-art algorithm (RB), often does not produce partitions of good quality. Here, we investigate the behavior of $\mathrm{RB}$ under this constraint and we present a comparison between $\mathrm{RB}$ and the two main alternatives for such problems. More precisely, we compare the KGGGP method that have been previously proposed by the authors and a new implementation of the algorithm used in $\mathrm{KPATOH}$ (named RBBGM). This implementation is necessary for a systematic comparison of the algorithms that handle fixed vertices since $\mathrm{KPATOH}$ is not publicly available.

In this study, KGGGP and RBBGM are tested on two different configurations of fixed vertices and their results in terms of edgecut minimization and runtime performance indicate that $\mathrm{RB}$ is not the optimal solution for such problems. More precisely, in the first experiment KGGGP and RBBGM exhibit a maximum edgecut gain of $40 \%$ and $30 \%$, respectively, compared to Sсотсн. In the second experiment KGGGP has a maximum gain of $26 \%$ and $35 \%$ compared to SCOTCH and RBBGM, respectively. Finally note that KGGGP remains robust to different graph structures which is not the case for ScOTCH or RBBGM, but is susceptible to an increasing number of parts in terms of runtime performance.

Among the perspectives of this work is to apply the KGGGP algorithm for load-balancing of complex multi-physics simulations. In such configurations fixed vertices may influence the partitioning results between different physical components improving the overall partitioning quality of the solution.

\section{Acknowledgment}

The authors would like to thank Bora Uçar for his valuable advice for the implementation of the RBBGM method used in this study. 


\section{References}

[1] M. Predari, A. Esnard, A k-way greedy graph partitioning with initial fixed vertices for parallel applications, in : 24th Euromicro International Conference on Parallel, Distributed, and Network-Based Processing, PDP 2016, Heraklion, Crete, Greece, February 17-19, 2016, 2016, pp. 280-287.

[2] J. D. Teresco, K. D. Devine, J. E. Flaherty, Partitioning and dynamic load balancing for the numerical solution of partial differential equations, in : Numerical Solution of Partial Differential Equations on Parallel Computers, Vol. 51, 2006, pp. 55-88.

[3] N. J. Dingle, P. G. Harrison, W. J. Knottenbelt, Uniformization and hypergraph partitioning for the distributed computation of response time densities in very large markov models, J. Parallel Distrib. Comput. 64 (8) (2004) 908-920.

[4] B. Hendrickson, R. W. Leland, R. V. Driessche, Enhancing data locality by using terminal propagation., in : HICSS (1), 1996, pp. 565-574.

[5] B. Hendrickson, R. W. Leland, R. V. Driessche, Skewed graph partitioning, in : Eighth SIAM Conference on Parallel Processing for Scientific Computing, 1997.

[6] C. Aykanat, B. B. Cambazoglu, F. Findik, T. Kurc, Adaptive decomposition and remapping algorithms for object-space-parallel direct volume rendering of unstructured grids, J. Parallel Distrib. Comput. 67 (2007) 77-99.

[7] U. V. Catalyurek, E. G. Boman, K. D. Devine, D. Bozdă̆, R. T. Heaphy, L. A. Riesen, A repartitioning hypergraph model for dynamic load balancing, J. Parallel Distrib. Comput. 69 (8) (2009) 711-724. 
[8] S. Plimpton, S. Attaway, B. Hendrickson, J. Swegle, C. Vaughan, D. Gardner, Parallel transient dynamics simulations, J. Parallel Distrib. Comput. 50 (1) (1998) 104-122.

[9] K. Brown, S. Attaway, S. Plimpton, B. Hendrickson, Parallel strategies for crash and impact simulations, Computer Methods in Applied Mechanics and Engineering 184 (2-4) (2000) 375-390.

[10] C. Walshaw, M. Cross, K. McManus, Multiphase mesh partitioning, Applied Mathematical Modelling 25 (2) (2000) 123 - 140, dynamic load balancing of mesh-based applications on parallel.

[11] M. Predari, A. Esnard, Coupling-aware graph partitioning algorithms : Preliminary study, in : 21st International Conference on High Performance Computing, HiPC 2014, Goa, India, December 17-20, 2014, 1-10, 2014.

[12] G. Karypis, V. Kumar, Multilevel algorithms for multi-constraint graph partitioning, in : Proceedings of the 1998 ACM/IEEE Conference on Supercomputing, SC '98, IEEE Computer Society, Washington, DC, USA, 1998, pp. 1-13.

[13] G. Karypis, Multi-constraint mesh partitioning for contact/impact computations, in : Proceedings of the 2003 ACM/IEEE Conference on Supercomputing, SC '03, ACM, New York, NY, USA, 2003, pp. 56-.

[14] P. Maria, Load balancing for parallel coupled simulations, Ph.D. thesis, University of Bordeaux (2016).

[15] A. E. Caldwell, A. B. Kahng, A. A. Kennings, I. L. Markov, Hypergraph partitioning for VLSI CAD : Methodology for heuristic development, experimentation and reporting, in : Proceedings of the 36th Annual ACM/IEEE Design Automation Conference, DAC '99, 1999, pp. 349-354. 
[16] A. E. Dunlop, B. W. Kernighan, A procedure for placement of standard-cell VLSI circuits., IEEE Trans. on CAD of Integrated Circuits and Systems 4 (1) (1985) 92-98.

[17] C. Aykanat, B. B. Cambazoglu, B. Uçar, Multi-level direct k-way hypergraph partitioning with multiple constraints and fixed vertices, J. Parallel Distrib. Comput. 68 (2008) 609-625.

[18] B. Hendrickson, T. G. Kolda, Graph partitioning models for parallel computing, Parallel Comput. 26 (12) (2000) 1519-1534.

[19] M. R. Garey, D. S. Johnson, Computers and Intractibility : A Guide to the Theory of NP-Completeness, W. H. Freeman, 1979.

[20] B. Hendrickson, R. Leland, An improved spectral graph partitioning algorithm for mapping parallel computations, SIAM J. Sci. Comput. 16 (2).

775 [21] R. Leland, B. Hendrickson, A multilevel algorithm for partitioning graphs, in : 1995 ACM/IEEE conference on Supercomputing, 1995.

[22] G. Karypis, V. Kumar, Multilevel k-way partitioning scheme for irregular graphs, Journal of Parallel and Distributed Computing 48 (1998) 96-129.

[23] B. W. Kernighan, S. Lin, An efficient heuristic procedure for partitioning graphs, Bell System Technical Journal 49 (1970) 291-307.

[24] C. M. Fiduccia, R. M. Mattheyses, A linear-time heuristic for improving network partitions, 19th Design Automation Conference (1982) 175-181.

[25] G. Karypis, METIS, HMETIS, PARMETIS, http://glaros.dtc.umn. edu/gkhome/metis.

${ }_{785}{ }_{[26]}$ F. Pellegrini, SCOTCH, http://www.labri.fr/perso/pelegrin/ scotch/. 
[27] P. Sanders, C. Schulz, Think Locally, Act Globally : Highly Balanced Graph Partitioning, in : Proceedings of the 12th International Symposium on Experimental Algorithms (SEA'13), Vol. 7933 of LNCS, Springer, 2013, pp. $164-175$.

[28] U. V. Catalyurek, C. Aykanat, PaToH : A Multilevel Hypergraph Partitioning Tool, http://bmi.osu.edu/ umit/software.html (1999).

[29] Zoltan : Parallel partitioning, load balancing and data-management services, http://www.cs.sandia.gov/Zoltan/Zoltan.html.

[30] B. Vastenhouw, R. H. Bisseling, A two-dimensional data distribution method for parallel sparse matrix-vector multiplication, SIAM Rev. 47 (1) (2005) 67-95.

[31] H. D. Simon, S.-H. Teng, How good is recursive bisection?, SIAM J. Sci. Comput 18 (1995) 1436-1445.

[32] H. W. Kuhn, The hungarian method for the assignment problem, Naval Research Logistics Quarterly 2 (1-2) (1955) 83-97.

[33] J. Ciarlet, P., F. Lamour, On the validity of a front-oriented approach to partitioning large sparse graphs with a connectivity constraint, Numerical Algorithms 12 (1) (1996) 193-214.

[34] R. Battiti, A. Bertossi, Differential greedy for the 0-1 equicut problem, in : in Proceedings of the DIMACS Workshop on Network Design : Connectivity and Facilities Location, American Mathematical Society, 1997, pp. 3-21.

[35] D. Bader, H. Meyerhenke, P. Sanders, C. Schulz, A. Kappes, D. Wagner, Benchmarking for graph clustering and partitioning, in : R. Alhajj, J. Rokne (Eds.), Encyclopedia of Social Network Analysis and Mining, Springer New 
York, 2014, pp. 73-82.

URL http://www.cc.gatech .edu/dimacs10

[36] R. Diekmann, R. Preis, F. Schlimbach, C. Walshaw, Shape-optimized mesh partitioning and load balancing for parallel adaptive FEM, Parallel Computing 26 (12) (2000) 1555-1581.

[37] S. Rajamanickam, E. G. Boman, Parallel partitioning with zoltan : Is hypergraph partitioning worth it?, in : Graph Partitioning and Graph Clustering, 10th DIMACS Implementation Challenge Workshop, Georgia Institute of Technology, Atlanta, GA, USA, February 13-14, 2012. Proceedings, 2012, pp. $37-52$. 
TABLE 5: List of graphs used for experiments from the popular DIMACS'10 collection.

\begin{tabular}{|c|c|c|c|c|c|c|c|}
\hline \multirow[b]{2}{*}{ group } & \multirow[b]{2}{*}{ collection } & \multirow[b]{2}{*}{ graph } & \multirow[b]{2}{*}{$\#$ vtx } & \multirow[b]{2}{*}{ \# edges } & \multicolumn{3}{|c|}{ degree } \\
\hline & & & & & avg & $\min$ & $\max$ \\
\hline 1 & walshaw & 144 & 144,649 & $1,074,393$ & 14.86 & 4 & 26 \\
\hline 1 & walshaw & 4elt & 15,606 & 45,878 & 5.88 & 3 & 10 \\
\hline 1 & walshaw & $\operatorname{cs} 4$ & 22,499 & 43,858 & 3.90 & 2 & 4 \\
\hline 1 & walshaw & cti & 16,840 & 48,232 & 5.73 & 3 & 6 \\
\hline 1 & walshaw & fe_4elt2 & 11,143 & 32,818 & 5.89 & 3 & 12 \\
\hline 1 & walshaw & fe_ocean & 143,437 & 409,593 & 5.71 & 1 & 6 \\
\hline 1 & walshaw & fe_sphere & 16,386 & 49,152 & 6.00 & 4 & 6 \\
\hline 1 & walshaw & whitaker3 & 9,800 & 28,989 & 5.92 & 3 & 8 \\
\hline 1 & walshaw & wing & 62,032 & 121,544 & 3.92 & 2 & 4 \\
\hline 1 & walshaw & auto & 448,695 & $3,314,611$ & 14.77 & 4 & 37 \\
\hline 1 & matrix & audikw1 & 943,695 & $38,354,076$ & 81.28 & 20 & 344 \\
\hline 1 & matrix & ecology1 & $1,000,000$ & $1,998,000$ & 4.00 & 2 & 4 \\
\hline 1 & matrix & thermal2 & $1,227,087$ & $3,676,134$ & 5.99 & 2 & 10 \\
\hline 1 & matrix & af_shell10 & $1,508,065$ & $25,582,130$ & 33.93 & 14 & 34 \\
\hline 1 & matrix & G3_circuit & $1,585,478$ & $3,037,674$ & 3.83 & 1 & 5 \\
\hline 1 & matrix & nlpkkt120 & $3,542,400$ & $46,651,696$ & 26.34 & 4 & 27 \\
\hline 1 & matrix & ldoor & 952,203 & $22,785,136$ & 47.86 & 27 & 76 \\
\hline 1 & matrix & cage15 & $5,154,859$ & $47,022,346$ & 18.24 & 2 & 46 \\
\hline 1 & numerical & NACA0015 & $1,039,183$ & $3,114,818$ & 5.99 & 3 & 10 \\
\hline 1 & numerical & 333SP & $3,712,815$ & $11,108,633$ & 5.98 & 2 & 28 \\
\hline 1 & numerical & NLR & $4,163,763$ & $12,487,976$ & 6.00 & 3 & 20 \\
\hline 1 & numerical & adaptive & $6,815,744$ & $13,624,320$ & 4.00 & 2 & 4 \\
\hline 1 & numerical & AS365 & $3,799,275$ & $11,368,076$ & 5.98 & 2 & 14 \\
\hline 1 & numerical & M6 & $3,501,776$ & $10,501,936$ & 6.00 & 3 & 10 \\
\hline 1 & numerical & channel-b050 & $4,802,000$ & $42,681,372$ & 17.78 & 6 & 18 \\
\hline 1 & numerical & venturiLevel3 & $4,026,819$ & $8,054,237$ & 4.00 & 2 & 6 \\
\hline 1 & dynframe & hugebubbles-00000 & $18,318,143$ & $27,470,081$ & 3.00 & 2 & 3 \\
\hline 1 & dynframe & hugebubbles-00010 & $19,458,087$ & $29,179,764$ & 3.00 & 2 & 3 \\
\hline 1 & dynframe & hugebubbles-00020 & $21,198,119$ & $31,790,179$ & 3.00 & 2 & 3 \\
\hline 1 & dymframe & hugetrace-00000 & $4,588,484$ & $6,879,133$ & 3.00 & 2 & 3 \\
\hline 1 & dymframe & hugetrace-00010 & $12,057,441$ & $18,082,179$ & 3.00 & 2 & 3 \\
\hline 1 & dynframe & hugetrace-00020 & $16,002,413$ & $23,998,813$ & 3.00 & 2 & 3 \\
\hline 1 & dynframe & hugetric-00000 & $5,824,554$ & $8,733,523$ & 3.00 & 2 & 3 \\
\hline 1 & dynframe & hugetric-00010 & $6,592,765$ & $9,885,854$ & 3.00 & 2 & 3 \\
\hline 2 & clustering & citationCiteseer & 268,495 & $1,156,647$ & 8.62 & 1 & 1318 \\
\hline 2 & clustering & coAuthorsCiteseer & 227,320 & 814,134 & 7.16 & 1 & 1372 \\
\hline 2 & clustering & coAuthorsDBLP & 299,067 & 977,676 & 6.54 & 1 & 336 \\
\hline 2 & clustering & coPapersCiteseer & 434,102 & $16,036,720$ & 73.88 & 1 & 1188 \\
\hline 2 & clustering & coPapersDBLP & 540,486 & $15,245,729$ & 56.41 & 1 & 3299 \\
\hline 2 & clustering & as-22july06 & 22,963 & 48,436 & 4.22 & 1 & 2390 \\
\hline 2 & streets & asia & $11,950,757$ & $12,711,603$ & 2.13 & 1 & 9 \\
\hline 2 & streets & belgium & $1,441,295$ & $1,549,970$ & 2.15 & 1 & 10 \\
\hline 2 & streets & germany & $11,548,845$ & $12,369,181$ & 2.14 & 1 & 13 \\
\hline 2 & streets & great-britain & 7733822 & 8156517 & 2.11 & 1 & 8 \\
\hline 2 & streets & italy & $6,686,493$ & $7,013,978$ & 2.10 & 1 & 9 \\
\hline 2 & streets & netherlands & $2,216,688$ & $2,441,238$ & 2.20 & 1 & 7 \\
\hline 2 & streets & luxembourg & 114,599 & 119,666 & 2.09 & 1 & 6 \\
\hline
\end{tabular}

